\title{
Lungensequester als Ursache rezidivierender Pneumonien
}

\author{
Bronchopulmonary Sequestration as a Cause for Recurrent Pneumonia
}

Autoren

Institute
K. Schreiner ${ }^{1}$, C. Cornelissen ${ }^{1}$, D. Frechen ${ }^{1}$, J. Spillner ${ }^{2}$, S. Krüger ${ }^{1}$

Medizinische Klinik I, Universitätsklinikum Aachen

2 Klinik für Herz- und Thoraxchirurgie, Universitätsklinikum Aachen eingereicht 16.11.2010

akzeptiert 19.11.2010

\section{Bibliografie}

Dol http://dx.doi.org/

10.1055/s-0030-1256050

Online-Publikation: 22. 12. 2010

Pneumologie 2011; 65:

37-38 @ Georg Thieme

Verlag KG Stuttgart · New York

ISSN 0934-8387

Korrespondenzadresse

Priv. Doz. Dr. med.

Stefan Krüger

Medizinische Klinik I

Universitätsklinikum RWTH

Aachen

Pauwelsstr. 30

52057 Aachen

stkrueger@ukaachen.de
Eine 23-jährige Patientin stellte sich mit rezidivierenden Pneumonien in der pneumologischen Sprechstunde vor. In den letzten 18 Monaten war es viermal zu einer Pneumonie des rechten Unterlappens gekommen. Nach Antibiotikatherapie war die Pneumonie jeweils komplikationslos vollständig ausgeheilt.

Bei der aktuellen Vorstellung war die Patientin beschwerdefrei. Hinweise für eine angeborene oder erworbene Immunschwäche ergaben sich nicht. Der körperliche Untersuchungsbefund zeigte eine junge und gesund erscheinende Patientin in sehr gutem Allgemein- und normalen Ernährungszustand. Keine Dauermedikation, kein inhalatives Zigarettenrauchen, gelegentlicher Alkoholkonsum. Keine relevanten Vorerkrankungen, familiäre Anamnese und Berufsanamnese ohne
Besonderheiten. Die kapilläre Blutgasanalyse und der Lungenfunktionstest waren unauffällig. Im Labor keine Auffälligkeiten, insbesondere kein Hinweis auf eine Infektion oder Immunschwäche. Die Röntgen-Thorax-Untersuchung zeigte unspezifische Veränderungen im rechten Unterfeld nach stattgehabter Pneumonie zuletzt vor drei Wochen ohne flächenhaftes Infiltrat $(\bullet$ Abb. 1).

Aufgrund der rezidivierenden Pneumonien führten wir eine kontrastmittelgestützte CT- Untersuchung des Thorax durch. Diese erbrachte den Nachweis aberranter Arterien, welche aus der Aorta abdominalis entsprangen und den rechten Unterlappen versorgten ( $\bullet$ Abb. 2, 3).

Bronchoskopisch war der Befund inklusive der entnommenen Sekrete aus dem rechten Unterlappen unauffällig. Bei V.a. einen Lungenseques-
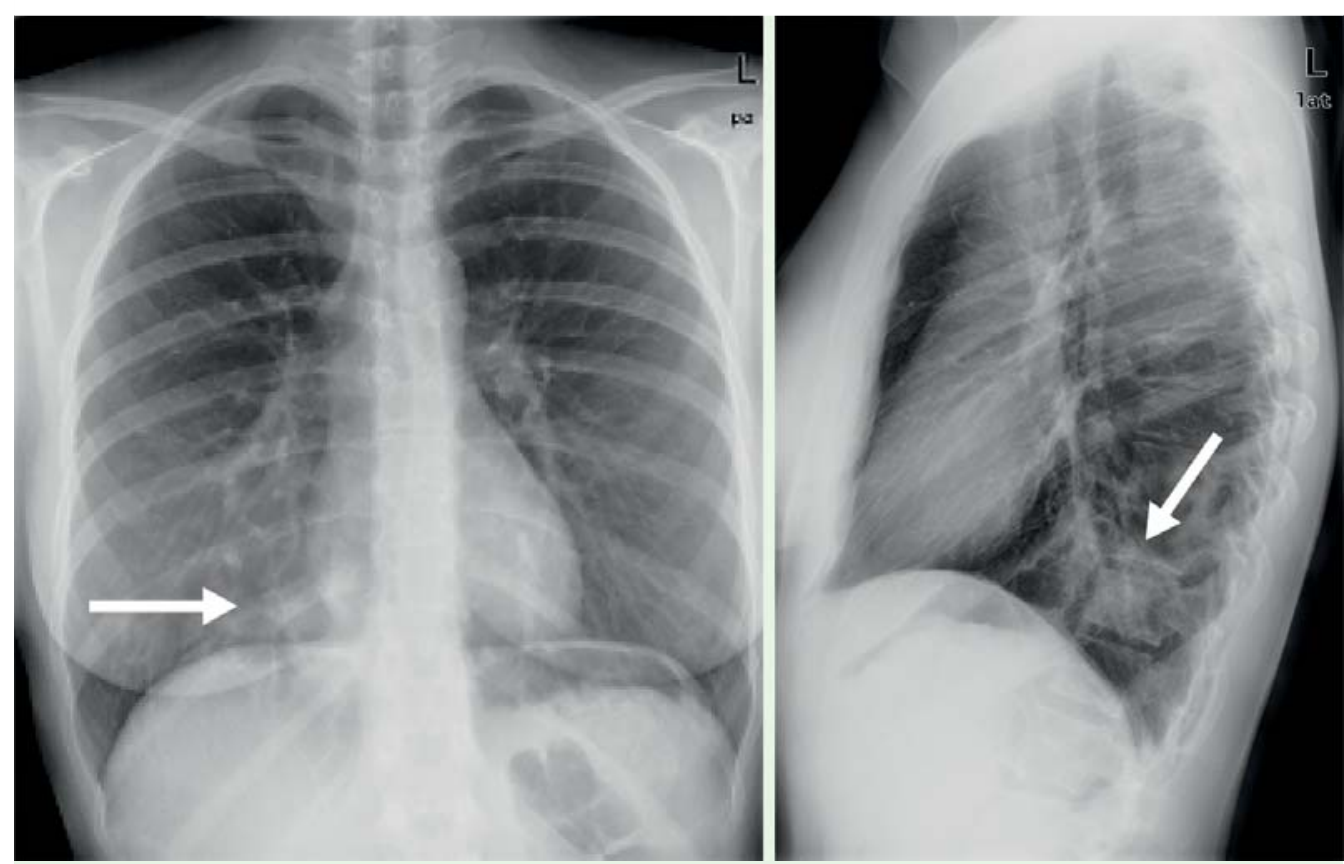

Abb. 1 Röntgen Thorax in 2 Ebenen mit postpneumonischen Veränderungen (Pfeile) im rechten Unterfeld. 

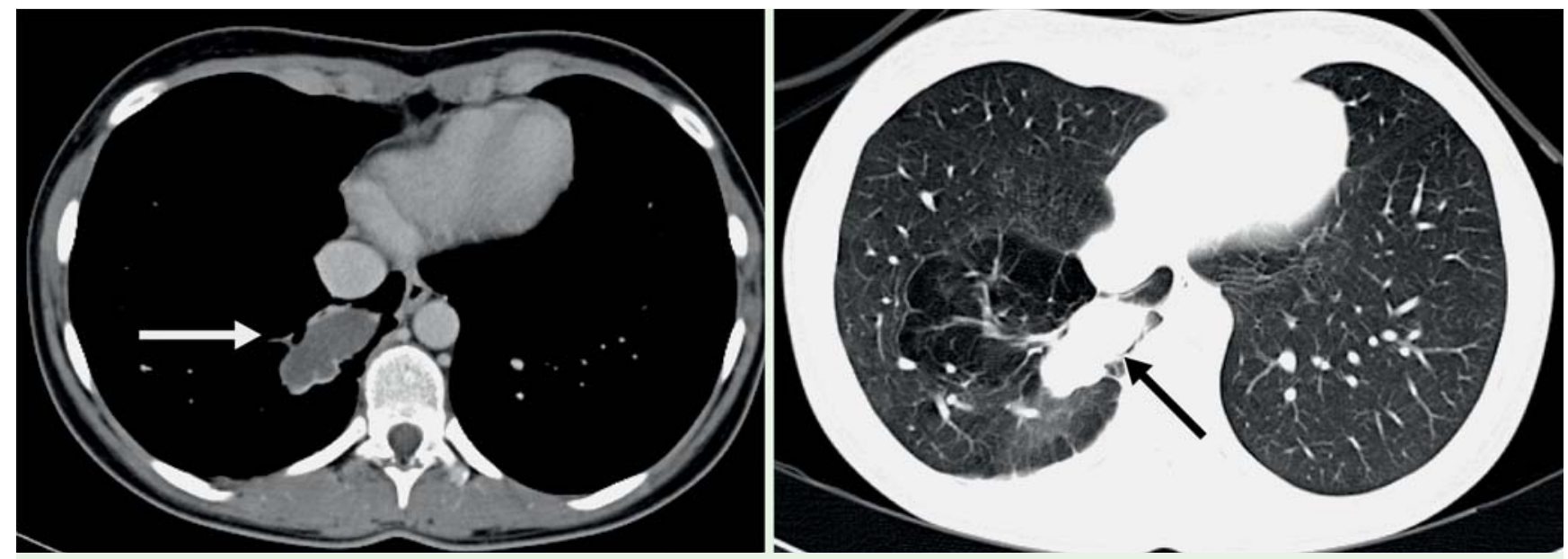

Abb. 2 In der kontrastmittelgestützten Computertomografie des Thorax sieht man zystisch imponierende und emphysematöse Veränderungen (Pfeile) im rechten Unterlappen nach wiederholten Pneumonien.

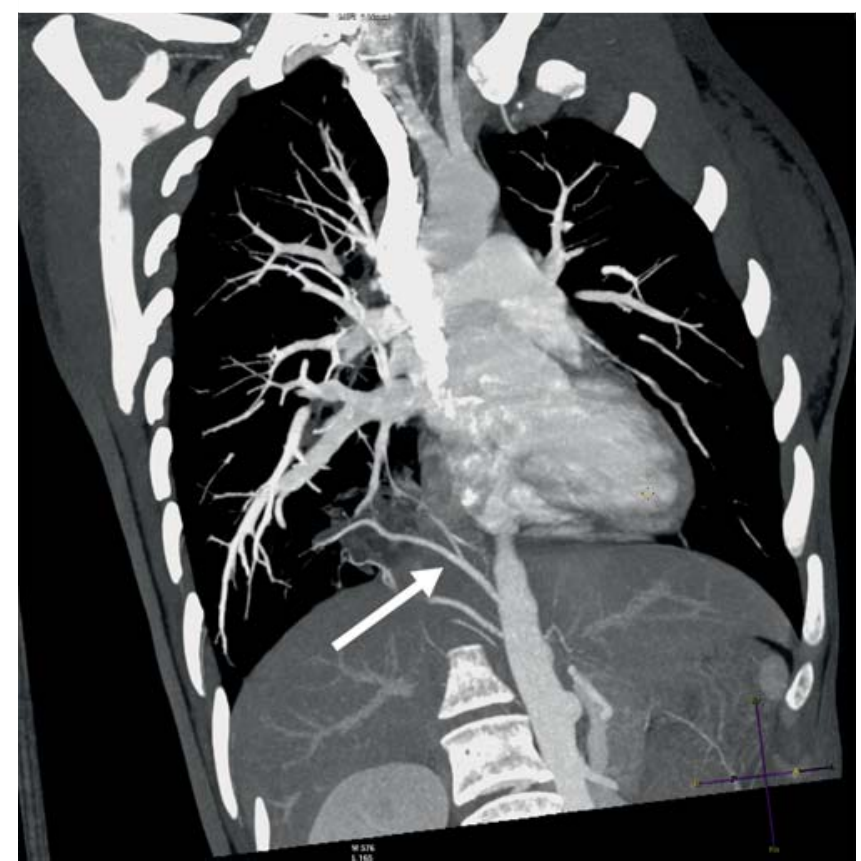

Abb. 3 Die Computertomografie des Thorax zeigt die aberranten Arterien (Pfeil), die direkt der Aorta abdominalis entspringen und den Sequester im rechten Unterlappen versorgen.

ter wurde die Patientin thoraxchirurgisch vorgestellt. Es erfolgte komplikationslos die atypische Resektion eines Sequesters des rechten Unterlappens. Im folgenden Jahr der Nachbeoachtung traten keine Pneumonien mehr auf.

Man unterscheidet intralobäre (in der Lunge liegende, umgeben von gemeinsamer Pleura) von extralobären (außerhalb des funktionellen Lungenparenchyms liegende, mit eigener Pleura überzogen) Lungensequester. Die häufigste Form ist mit $75 \%$ aller Fälle der intralobäre Lungensequester, wie auch in unserem Fall. Diese finden sich vor allem in den Unterlappen. Extralobäre Sequester sind vor allem links basal lokalisiert. Eine Übersicht gibt $\bigcirc$ Tab. 1. Die Therapie der Wahl ist in beiden Fällen die chirurgische Resektion.

Lungensequester sind kongenitale Fehlbildungen der Lunge, die auf einer Störung in der Embryogenese mit einer überzähligen Lungenknospe beruhen. Die Sequester haben keinen primären
Tab. 1 Charakteristika der extra- und intralobären Lungensequester.

\begin{tabular}{|c|c|c|}
\hline & $\begin{array}{l}\text { extralobärer } \\
\text { Sequester }\end{array}$ & $\begin{array}{l}\text { intralobärer } \\
\text { Sequester }\end{array}$ \\
\hline Alter bei & meist & meist ab \\
\hline Diagnosestellung & 1. Lebensjahr & 20. Lebensjahr \\
\hline Symptome & $\begin{array}{l}\text { meist Symptome im } \\
\text { 1. Lebensjahr, Dyspnoe, } \\
\text { Zyanose, gestörtes } \\
\text { Essverhalten }\end{array}$ & $\begin{array}{l}\text { wiederholte Pneumo- } \\
\text { nien in gleicher Lokali- } \\
\text { sation, Hämoptysen, } \\
\text { meist Zufallsbefund }\end{array}$ \\
\hline Häufigkeit & $25 \%$ & $75 \%$ \\
\hline Lokalisation & $\begin{array}{l}\text { linke Lunge para- } \\
\text { vertebral }(90 \%)\end{array}$ & $\begin{array}{l}\text { posterobasaler Unter- } \\
\text { lappen ( } 95 \%), \\
\text { häufiger links }\end{array}$ \\
\hline $\begin{array}{l}\text { arterielle } \\
\text { Gefäßversorgung }\end{array}$ & $\begin{array}{l}\text { meist zahlreiche klei- } \\
\text { nere Arterien aus der } \\
\text { Aorta }(80 \%)\end{array}$ & $\begin{array}{l}\text { meist ein großkalibri- } \\
\text { ges Gefäß aus der } \\
\text { Aorta }(73 \%)\end{array}$ \\
\hline venöser Abfluss & Vena cava inferior ( $80 \%)$ & Lungenvenen (95\%) \\
\hline Anatomie Pleura & $\begin{array}{l}\text { eigene Pleura viszeralis, } \\
\text { hierdurch vom übrigen } \\
\text { Lungengewebe getrennt }\end{array}$ & $\begin{array}{l}\text { gemeinsame viszerale } \\
\text { Pleura innerhalb eines } \\
\text { Lungenlappens }\end{array}$ \\
\hline $\begin{array}{l}\text { Geschlechter- } \\
\text { verhältnis }\end{array}$ & ca. 80 \% männlich & $1: 1$ \\
\hline
\end{tabular}

Anschluss an den Tracheobronchialbaum und werden von aberranten arteriellen Gefäßen meist aus der Aorta versorgt. Es handelt sich um eine seltene Fehlbildung, die 0,15-6\% aller pulmonalen Fehlbildungen ausmacht. Rezidivierende pulmonale Infektionen wie Pneumonien können Folge eines Lungensequesters sein, sie können aber auch selbst zur Entwicklung von intralobären Lungensequestern führen.

\section{Interessenkonflikt}

$\nabla$

Die Autoren geben an, dass kein Interessenkonflikt besteht. 